\title{
Product hacking as a systematic intervention: towards new strategies and platforms in design education
}

\author{
Humanur Bagli ${ }^{1}$ (D) . Tugce Ecem Tufek Serifoglu ${ }^{2}$
}

Accepted: 7 July 2021 / Published online: 5 August 2021

(C) The Author(s), under exclusive licence to Springer Nature B.V. 2021

\begin{abstract}
This paper is mainly about how product hacking can be categorized and implemented in a systematic way in design education. The theoretical or analytical part of the project is based on a study in which a great number of hacking projects are reviewed and categorized found in popular DIY platforms. The implementation or synthesis part of the research is a design project held in an industrial design department with freshman design students based on the categorization of function proposed, which are namely; enhance, change and add function. In this project students are required to make three kinds of interventions on functions of existing products, also share their projects in the selected platform (www.instr uctables.com), so that other people can find, criticize, develop, or apply it by using the instructions given. Sharing in platforms like these creates a connection between the analytical and synthetic phases of the paper, and also makes it different from other classical projects confined to a closed studio atmosphere.This paper also seeks for the possibility of fresh perspectives on issues like sustainability, ready-made and open source design by engaging students in an intervention-based design process where they look for formulating their designs in a methodological way.One other aspect of the project was the idea that it has the potential to create an advantage or value for the time and context it was given, that is when the COVID-19 has started to influence our lives deeply.
\end{abstract}

Keywords Design education · Product hacking · Intervention · DIY platforms · COVID$19 \cdot$ Sustainability

Humanur Bagli

humanur@gmail.com

Tugce Ecem Tufek Serifoglu

tecemtufek@gmail.com

1 Faculty of Architecture and Design, Department of Industrial Design, Marmara University, Istanbul, Turkey

2 Department of Industrial Design, Istanbul Bilgi University, Istanbul, Turkey 


\section{Introduction}

This paper is an attempt to suggest a model in design studio education by presenting a studio project in the first year design studio based on the idea of hacking or intervention. One original aspect of the project is the platforms or the arena such as instructables.com, where the projects are shared with different people and parties other than only the classmates and tutors.

The project tries to implement findings of a research about product hacking in a design studio for the sake of design education. In the mentioned research study (Tüfek, 2019) an extensive number of product hacking projects are reviewed and categorized based on three most popular DIY platforms in which product hacking projects are exhibited in detail, including instructables.com, ikeahackers.com and Makezine. The implementation or synthesis part of the research, on the other hand, is based on a studio project held in an industrial design department that will be explained and exemplified in this paper.

Although product hacking is a relatively novel concept, we can encounter examples of functional interventions to the existing products under other known trends such as maker movement and DIY (Saakes, 2009; Wolf \& McQuitty, 2011). Despite its novelty, there are also cases where the hacking mindset is employed in the education context by advocating alternative approaches to the existing curricula such as School Retool, Hacker High School (Duman, 2020). In the art education setting, Lewis and Thurman (2019) investigate the form and function relationship rather philosophically through hacking and tinkering activities. When it comes to industrial design education, we can say that it is much easier to see a more organic connection between hacking practices of existing objects and product design. Moreover, application of these practices to industrial design curricula could have many practical outcomes, like increasing technological agility, encouraging cooperation, bridging professional and daily contexts and cultivating student creativity (Duman, 2020).

Suggesting a new stance in design studio education by presenting a first year design studio project, the paper aims to trigger questions and discussions from various perspectives. Some of these questions can be counted as;

- How can a systematic categorization based on a field research made on digital platforms can be implemented as a basis of a design education project?

- How to raise awareness about terminology related with design such as sustainability, recycling and upcycling, especially in the first year design studio by practice in a methodological way?

- How can design education contribute to extreme social cases and contexts like COVID19 ?

- What can be the alternative grounds of design ideas and outcomes and the influences to be shared with different parties like makers, DIY people, hobbyists, entrepreneurs, and other stakeholders?

- And last but not the least, how to consider and develop a project in a designerly way in a project formulation to be placed in such platforms.

First consideration of the paper is an attempt to tie a field research into a design project, which is Tufek's study here (2019); in other words, questioning how the analysis based on hacking platforms can lead to build a unique structure of a brief for a design project.

Second discussion is about the importance of design brief as a formula with the potential to change the attitude of the students and tutors about some issues like reuse, recycling 
etc. in the process of design. It can be said that the design brief here is an example of how awareness can be raised about a certain terminology given in the process rather than imposed in the beginning.

Third discussion relates to hot circumstances like COVID-19, or other social crises that have a big impact on people and their lives. Design education can be regarded as agile enough about taking action in different societal issues that could and should be thought of and reformulated according to these kinds of situations. This kind of project formulation could also be an idea for a model useful for online design education, where the projects are shared and developed online with a big community. The advantage lies in the fact that even if the shared projects are purely tangible, there is no need for physical contact among the parties that fits well to the pandemic situation.

The fourth one is about the importance and potential of building relationships with different stakeholders. There have been different projects that build different collaborations such as SMEs, companies and startups etc. with design schools from freshman to senior, undergraduate to graduate levels in design education (Er \& Er, 2003; Goellner et al., 2010; Darabi \& Clark, 2012; Soyupak \& Bagli, 2019). In this one, projects are aimed to be shared and presented in an open digital platform called instructables.com as an alternative area of collaboration in design education. This platform is selected because the nature of the project brief reflects a characteristic that has an emphasis on steps of instructions to create something out of an existing object and also because the process of production and idea generated is much more important than the outcome of the design process in such a brief.

The last concern is to be designerly as a category where the projects developed were asked to be distinctive than the ones in the site instructables.com as a challenge, to be held with considerations about design such as functionality, consistent visual language, consistent scenario, material sensitiveness and reproducibility.

Looking deeper into the focus of the project, we can easily understand the scope of the study excludes the issues related to cosmetic sides of design such as form giving, styling, brand and product identity, also choice and use of the material from scratch; however still feeding these considerations indirectly.

\section{Background of the project}

The background of the project is based on an analysis of the strategies of product hacking or intervention. The studies and the practices about this subject are not new. People's interest in self-creation and product modification dates back to the 1950s and is categorized under the title of DIY (Smith, 2014). Toffler's (1984) "prosumer" and Campbell's (2005) "craft consumer" and von Hippel (2006) "user innovator" can be given as examples from different times of history to the actors who study this subject in relation with products and their production cycles.

Today, there is an increasing interest in hacking, DIY or maker projects in the world, also in the design field. This can be traced from the growing number of online communities such as instructables.com where these projects featured (Saakes, 2009). The motivation for engaging in product interventions may originate from an external factor such as limitations of mass produced goods in the presence of specific user needs, as well as internal factors like personal-fulfillment of creating things (Wolf \& McQuitty, 2011). Alongside these motivational indicators, other particular points are also effective in the recent enthusiasm on hacking, DIY and maker projects. Kuznetsov and Paulos (2010) group these factors 
under two fundamental categories; "easy access to and affordability of tools" and "the emergence of new sharing mechanisms".

Today, the accessibility to flexible production techniques such as desktop size 3D printers, vacuum formers and open source software is higher. Consequently, this enables every person to be involved in the design and manufacturing process of products without depending on any other mediator. The ability of intervention to the products, in accordance to one's needs and desires, also constitutes the motivation for engaging in domestic production processes (Mota, 2011). On the other hand, rising popularity of online platforms also plays a crucial role in increasing the number of people doing product hacking projects, since people may easily communicate and collaborate freely through the web. Expanded attention towards these websites can be associated with the recent, well known Maker Movement which strongly promotes "sharing" and "participating" as its essential values (Hatch, 2014). It is a common thing for members of these platforms to share their projects step by step in detail and cooperate with and learn from each other on various projects.

These platforms where hacking is dominant also have some connections with the terms and tactics like recycle, reuse, upcycle, readymade etc. because the definition of hacking lies on the idea of working with the existing entities, whether it is software with the principles of coding, or objects with design decisions. That is why these issues are referred to this project and discussed during the process also because of their significance in the design literature.

The concept of hacking mainly belongs to the digital sphere and corresponds to unauthorized and backstage activities. That is why hacking, as a notion, expresses a strong sense of opposition. In physical space, a wide number of people utilize "hacking" for naming their creative uses and modifications of mass-produced products. In this sense, hacking gives to the user the power of "redefining" the products and brings people up to the same level with traditional authority figures in current production lines such as designers (Galloway et.al, 2004). In correlation, von Busch and Palmas (2006) define the practice of hacking as "breaking control" (p.37) and claim that hacking provides a more egalitarian approach in information distribution. Similarly, De Certeau's (1988) concepts of "tactics" and "strategies" are also notions which are generated to interpret this power struggle between authority and the others. According to him, the authorities have "strategies" which contain several plans and methods to realize their aims. Whereas the others have "tactics" which he fittingly describes as "an art of the weak", enabling the "weak" party to modify the current context according to their preferences. Although De Certeau constructs his "strategies" and "tactics" on a sociological level, his concept of "tactics" can be associated with the hacking practice itself in a way. Accordingly, this project can be restated as a way to empower the "weak" as De Certeau puts it with the power of design as a tool in the form of a strategy or a tactic. The term "weak" also applies for the position of the junior design students in design education, where they try to find their ways to become professionals by learning and developing strategies of their own discipline.

Hacking is a useful term that is close to intervention with rich references and examples of design. Even if hacking originally is a destructive tool to transform an existing system and more popular in digital production and defined as "circumventing a system's limitations to create new options", design hacking has a similar motivation as stated by Burnham. He says that design hacking as an ethos can be applied to our relationship with design "as a direct response to real problems faced by real people" (Burnham, 2019).

Burnham explains the positive sides of hacking as it "creates new realities, options and possibilities from those we are given, whether commercial, social or civic. It offers forth the notion of a democratization of design, by enabling the end user to be part of the 
process and not only on the receiving end of it. There is a triumphant message of individual resourcefulness and direct engagement when a hacker sensibility is applied" (Burnham, 2019).

In the project brief of this study, intervention is used as the keyword because it sounds more embracing as a term having more productive or positive denotations than hacking, even if it is less referred to in design literature, also because it is a more general term that makes it harder and challenging to find examples in a keyword search, so that students could find their own ways during the project development process. On the other hand, all these terms including hacking, DIY, maker movement, recycle, upcycle and reuse refers to a similar culture or attitude towards design, production, relationship with technology and society.

Intervention is defined in the brief of the project quoted from a dictionary definition as "a situation in which someone becomes involved in a particular issue, problem etc. in order to influence what happens" ("Intervention." n.d.) We can broadly say that intervention includes all kinds of involvement seeking to make a change. This term is used mostly for medical and educational systems where change is the ultimate goal. When we specifically start to talk about design intervention, it mostly refers to the changes in the real world, especially the material side of it, also sometimes design of services and experiences that can be intangible (Smith, 2019).

Smith (2019) defines design intervention as "prototypes that provoke real world action and intervene in human behavior" implying a deep effect on whom is the subject to this intervention. He also makes a comparison that is between art and design interventions. The example of "parking intervention" (Rebar parking day) he gives illustrates the difference between art and design interventions. In this example, the intervention is based on the double meaning of "park" and artists created a scene or performance in a car parking area in a city context where they behave as if this specialized area is for recreation just like in a green area. In this performance, intervention appears as a sort of happening or an art event which occurs in a certain time in the city life in order to give a certain message to the public, but actually no real "use" in life. We can say that it is an occasion that is put into parenthesis. In the definition Smith gives in his article on art intervention, the emphasis is on interacting with an existing structure or situation, an artwork, audience, or an institution or in the public domain ("Art Intervention", 2019) and implies no reference to use as design intervention, as suggested in this paper. So, design intervention in this project can be defined as something emphasizing the "function" side of a system with a more pragmatic approach, related also strongly with the pedagogic aims of a product design studio. In order to maintain such an aim, a categorization of hacking projects based on functionality is adopted and implemented on new objects as design interventions.

Actually, all design is intervention, because design is all about changing people's lives with material or systematic influences. One of the oldest definitions of design by Simon (1969) implies this in a very clear way: "Design is devising courses of action aimed at changing existing situations into preferred ones." Also one of the newest definitions of design by the World Design Organization is the one that broadens the scope of design from products to services and experiences: "...a strategic problem-solving process that drives innovation, builds business success, and leads to a better quality of life through innovative products, systems, services, and experiences." ("Definition of Industrial Design", 2020) Common point of this definition with Simon's is aiming for a better quality of life by exploring, finding out and implementing better interventions.

In this project, the principle of "interacting with an existing structure" as Smith suggests for intervention (2019), structure being the product chosen in this context is adopted, 


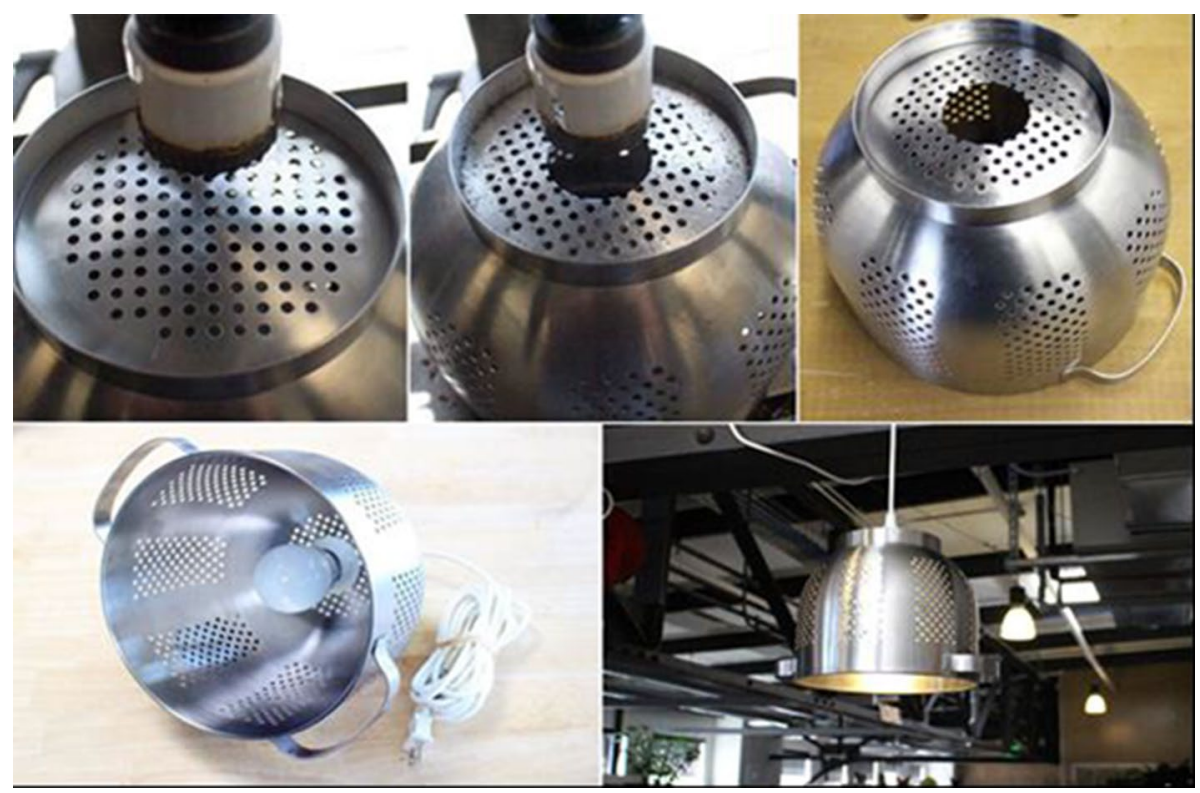

Fig. 1 Re-purposive hack: A colander is hacked into a lampshade ("IKEA HACK: Easy Lamps", n.d.)

however, with the aim of intervening to create a real advantage with the design tools, attitude and methods.

\section{The framework}

Tüfek $^{1}$ (2019) in her study, makes a categorization of product hacking based on the analysis of collaborative platforms such as Instructables, Makezine and IKEAHackers, namely Re-Purposive Hack, Augmentative Hack and Additive Hack. This categorization is the one that is selected for structuring the variations of products required from the students in the design brief of the project explained in this study. Because the chosen design hacking samples are from a big repertoire of digital sharing platforms such as Instructables, IKEAhackers.com and Makezine etc., the categorization is found useful for implementing as a design toolkit and implemented designs to be shared in similar platforms. We can say that the research resulted with this categorization defines the analytical part of the study; on the other hand, the implementation phase as a design project brief suggests a synthetic tool as a method to be tested in a similar platform.

Tüfek (2019) studies 300 hacking projects (uploaded between 2009 and 2018) from 3 different websites; Instructables, Ikeahackers and Makezine. The sample projects were chosen according to the functional changes made in the initial products. Thus, the study excluded the sole visual modifications. In the study, all of the data presented in the project pages, from tools and materials to step-by-step instructions, were examined

${ }_{1}^{1}$ A thesis supervised by the first author of this paper. 

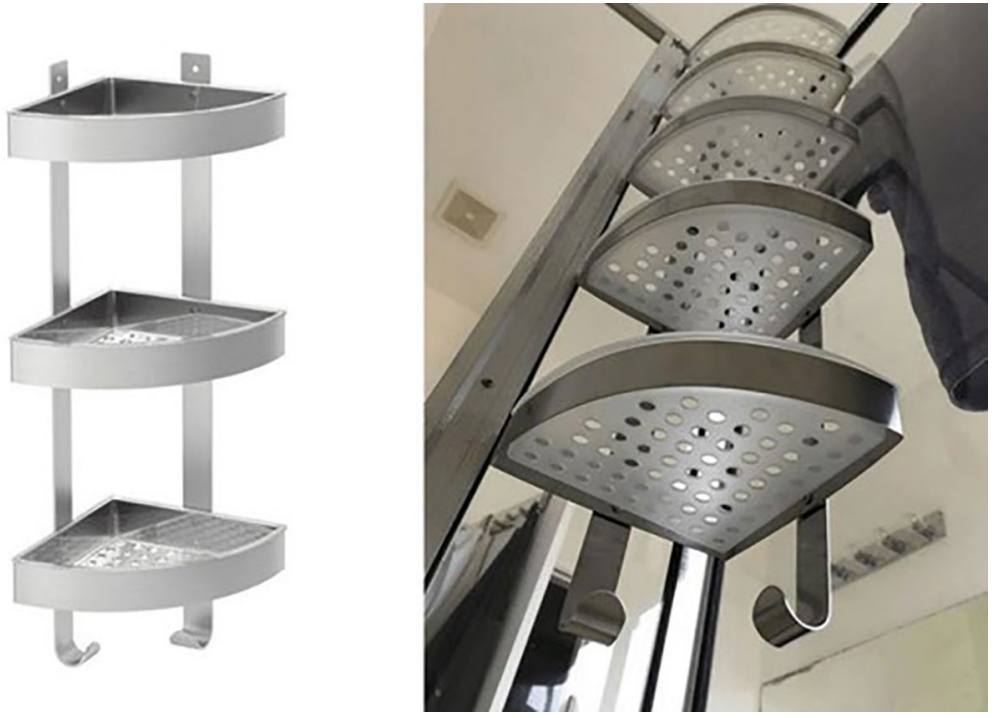

Fig. 2 Augmentative hack: Shower caddy before and after hacking ("Corner shower caddy: The Grundtal extra-long version”, 2018)
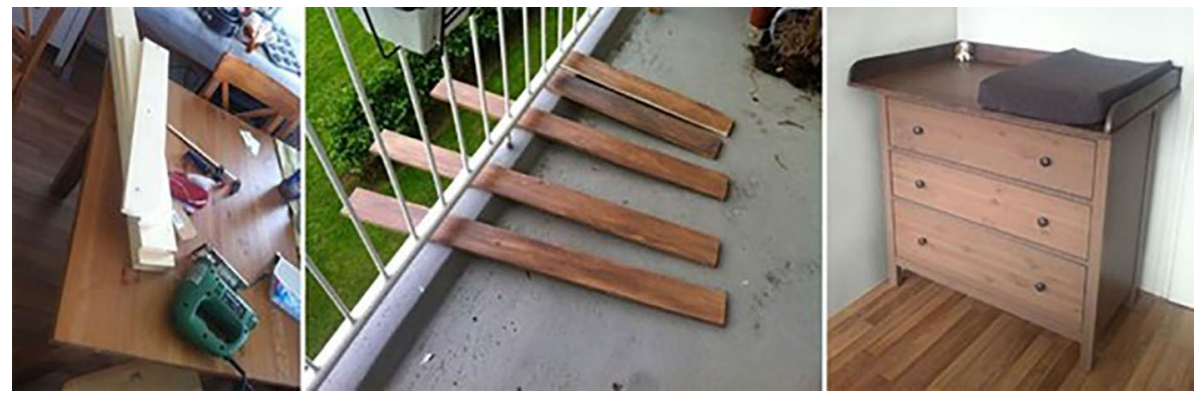

Fig. 3 Additive hack: Diaper change station is added on the dresser ("Hemnes Baby Changing DresserIKEA-Hack”, n.d.)

in the analyzing process. Departing from the functional changes in the products 3 categories were presented: Re-Purposive Hack, Augmentative Hack and Additive Hack. Tüfek (2019) reports, many of the projects in these platforms could be categorized as re-purposive hacks. These types of projects include a major change in the function of the hacked object, and the object no longer harbors its original function. A colander which is modified to be used as a lamp shade was given as an example for this category (Fig. 1). On the other hand, in augmentative hacking projects hackers aim to improve the original function of the product without changing or multiplying it. A hacking project which shows how to merge two showers' caddies for more storage area and easy installation was given as an exemplary case for augmentative hacks (Fig. 2). Finally, additive hacking projects simply aim to increase the number of functions that a product furnishes. Another hacking project which explains how to turn the top of a dresser into a 
The categorization in Túfek (2019)

Types of Product Hacking

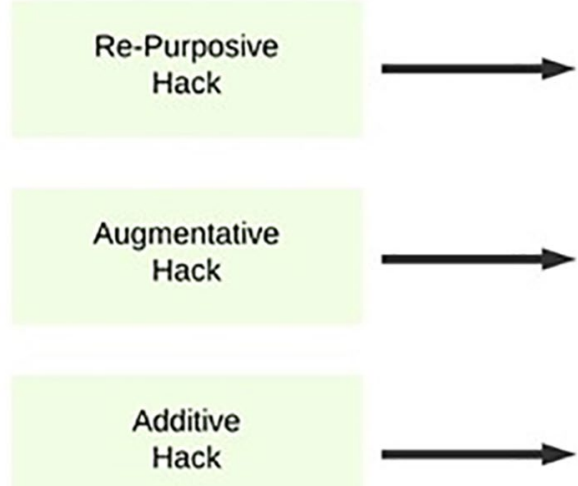

Transformed categorization used in 1st year Industrial Design Studio brief

\section{Product Intervention}

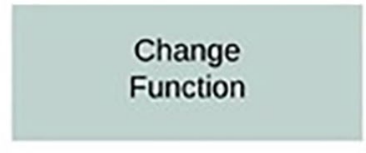

Enhance

Function

Add

Function

Fig. 4 The categorization of "types of product intervention" transformed from Tüfek (2019)

diaper change station by plainly setting up a bigger top and some railings (Fig. 3) can be given as an example for the additive hacking category.

There are of course other intervention types in these platforms that do not fit into these categories. As the study of Tufek is developed to inform and feed the design field from a different perspective, the types of projects especially where the functional strategies used were selected. This is also the reason why the same framework was applied as a categorization in the design studio project in this paper.

This categorization of Tüfek (2019) is selected for the systematization and a ground for variations of the project, namely Re-Purposive Hack, Augmentative Hack and Additive Hack. However, these names are redesignated as basic verbs for functions as the project is dependent on the functional variations. These are changing function for re-purposive, enhance function for augmentative and add function for additive hack (Fig. 4).

\section{Project and the design studio process}

Placing the design studio into the center, design education certainly has its own particular attributes. The unique characteristic of teaching design could be the result of the tacit nature of both design practice and accompanying knowledge. Polanyi (2009), as the creator of the concept, argues that some types of knowledge cannot be transferred or expressed via verbal communication. In a similar manner, Uluoğlu (2000) explains that one would simply learn designing through explanations if it was only about obtaining particular techniques. Furthermore, design studio provides an environment for students to gain lifelike experience by introducing them to hypothetical design problems (Tovey, 2015). Thus, they can grasp the implicit character of designing and also learn how to engage in a "reflective conversation" with the design process (Schön, 1984). 
Indeed, a freshman design student does not have the ability to "reflect-in-action" yet due to her lack of experience in designing (Schön, 1984). Because of that, for an inexperienced design student, starting from an existing object is more advantageous because they do not yet have experience with the methods and tools to develop and finalize a design object from scratch with the right choice of material and form. Starting with an existing object also makes it possible and easier to trace and assess the interventions as singled out parameters for the tutors and also for the sake of the research.

This project is formulated as the final project of a first year industrial design studio that was synchronic with the first days of the isolation time of COVID-19. Previous project in the semester was based on the use of corrugated cardboard and students had started to complain about the scarcity of the material and their difficulty of reaching the materials they needed. The final project was formulated according to this condition, and they were asked to start from existing objects they have in their homes. Students are required to take these steps presented in the brief:

Project: Intervention Design.

intervention: "a situation in which someone becomes involved in a particular issue, problem etc.

in order to influence what happens" https://www.macmillandictionary.com/dictionary/ british/intervention

In this project, you are required to choose one object that you have at home...

Make three alternatives to intervene that object to;

1. Enhance function

2. Change function

3. Add function

These interventions should be designerly... Your intervention should not exceed the original chosen object's characteristics, i.e. try to make as simple, less and smart interventions as possible for the most effective outcome. Also try to make interventions that are applicable for other people, i.e. instructable. (See www.instructables.com)

Think about how this intervention help people's lives in different ways for hard times like COVID-19.

\section{Requirements and Assessment Criteria for Final Jury:}

1. $1 / 1$ models

2. Storyboard about use scenario and the instructions

3. Technical Drawings

4. Design of the Logo and Presentation Boards

The area where students are supposed to address is based on the variables on functionality which is the main motivation of design. How can function be used as a variable in design is the domain to be played with and experimented in this project. It was decided to start with existing object/s chosen by the students so that they may have a chance to explore, analyze and intervene in this aspect of functionality. As the most characteristic aspect of this project, this critical choice of starting point also is 
effective in defining a pedagogic level where the freshmen design students could tackle as explained above.

This paper also seeks to sort of "naturalizing" or "internalizing" some terms like readymade, eco design, upcycling and recycling under the umbrella term sustainability in practice, rather than dry terminological entities to be researched on and implemented in the design process and on design pieces. So, we started the process by simply asking students to intervene on existing objects by finding out some strategies about function like adding, changing, and emphasizing.

As for the first year design students, the aim of the project is to come up with as many different ideas as possible, also report and visualize the process as steps rather than a perfect final product that was presented as a completed design piece. Because of the basic definition of the project, most of the outcomes are hardly the ones that can be produced and commercialized, rather, implemented as DIY pieces. So, the discussions based on the models (not sketches) which trigger above mentioned titles and strategies like readymade, recycle etc. becomes much more significant than the outcomes of the project itself.

However, another requirement related with the project does emphasize the outcomes of the project which is the step where they upload their projects to the site instructables.com. This step is significant from different perspectives: One is to carry a piece of design beyond the boundaries of the classroom and open it to another community where other makers, designers and DIY people share their ideas. Another significance is to open a design piece to a community where other parties can contribute to; a process which is totally different from a design process held in the classical design education process that ends with -sometimes- a harsh jury as a milestone event where only the members of design education are the members. Sharing a design idea with other parties in the platform before completion of the project and design as a process rather than an outcome is something rare in design paradigm, education and also in the design community. So, this attempt to centralize and highlight this episode of design makes it much closer to entrepreneurship ecosystems like incubation centers, pitch events and mentorship systems where failure is also embraced equally with success (Blank, 2011; Cantamessa et al., 2018; Wennberg et al., 2013).

One other aspect of the formulation of this project is the unexpected and extraordinary time and context of its delivery; it was given in the time when the COVID-19 had just started to sweep the world. One of the characteristics of this time was that students just like other people had difficulty to reach all the materials they needed to design and the only resource they could reach was the materials at home or nearby facilities. The project also seeks to instrumentalize this scarcity as an opportunity for design. This situation also presents new possibilities for the design ideas and products that are developed in this brief that can be implemented by other people thanks to the "instructable" nature of the project. It can also be stated as another advantage of the project. However, the interest towards online platforms, digital sharing and coworking were valid long before the pandemic, we can say its inevitable acceleration will affect this culture more in the future of design. So, the influence of pandemic is just a practical motivator for the shared project that is expected to lead to long term effects in formulation of such projects.

One drawback of the project is again stemming from the time and context where it was delivered. That is the lack of motivation of the students because of the radical change of the system of education, with no smooth transition from physical to digital or near to distant. This was also the case for the educators who tried to experience that transition in an effective and healthy way. Also, the fact that first year design students should experiment with 
real materials was also influential in this process. The real working models were obviously harder to criticize and help develop with online critique and juries.

Compared to the other projects that they dealt with during the semester based on the motivations like semantics of form giving, structural seating and table units out of cardboard; this project was odd in the sense that it started directly from existing objects. This initiation point is also what makes this project significant and original in its formulation; as all the discussions based on the notions like readymade, recycle, upcycle and eco-design have started almost naturally because of the unique structure of the brief.

So, based on these aspects, aims, and expected learning outcomes of the project could be summarized like this:

- To make students be critical about and work and design with existing objects and forms as sources of affordances and functional potentials.

- To make students released from the confined atmosphere of design studio, enable and empower them in other communities and non-authoritarian platforms; encourage them to cowork and codesign with such parties.

- Naturalizing terms like readymade, eco design, upcycling, and recycling by practicing them in the project formulation and their applications, not only as terminological entities.

- To make them understand that design is not just a form giving and cosmetic activity; but a systematic approach where all parameters related with scenario building, intervention and affordances are met.

\section{About the criteria of the project}

There is a series of design criteria in this project that has a mesh of connections to one another. Obviously the most dominant criteria is functionality as the main structure of the project brief is dependent on the variables of functionality; also functionality is the ultimate goal of design education, as the formula "form follows function" suggests. Regarding only this criterion, this project is very classical by questioning how this formulation is maintained in each design.

One of the other criteria is about the platforms that are going to be shared which are being instructable or definable. About this part of the project students are required to standardize each step of production so that other people who are interested in the project can make or reproduce that item, with suggestions of modifications or not. This brings forth the criteria of reproducibility which is also a very important part of industrial design practice and design education. We can say that this is also related with collaboration as the main motivation of sharing culture that is dominant in platforms mentioned and other important design paradigms like co-design and user centered design.

Another key criterion in this project sought is being designerly. The concern of being designerly is the one of which is totally common with the other projects held throughout the education of design. This criterion is sought for all kinds of different projects and able to be reached only by educational practice and expert critiques. This understanding of design is referenced to the "Design" that is written by capital D as Archer stated (1979). The reason why Design is written by capital D here is that it refers to a specialized body of knowledge and expertise and "collected experience of the material culture". This is what 
Cross (1982) mostly deals with when he mentions design discipline with its characteristics like being ill defined, solution focused, constructive, translating abstract into concrete and being aware of "design language".

In this project, all these parameters are hard to pinpoint on student projects, however cumulation of these parameters can be seen as outcomes with a kind of form and material sensitivity or consistency of visual language apart from the functionality per se. This sensitivity and its results as design pieces can be regarded as the core aim and concern of design education in general.

However, these concerns are the ones that distinguishes the projects developed in a design school by design students form the ones we see a platform like instructables.com, because this hard-to-achieve and abstract concern of being designerly is not the main issue in most of the items we see in platforms like this because most of the participants of these kind of platforms are not educated in design institutions with the concerns mentioned above. This situation refers to an ecosystem where the "democratization of design" is the subject of discussion (Fleischmann, 2015). So, this study can be seen as a first step to test whether the concern of being designerly makes a distinction among other items or contributes to what Fleischmann refers to as democratization of design in similar collective platforms.

Apart from the sensitivity to or consistency of material and form, consistency of scenario is another parameter peculiar to this and similar projects, that is its implicit relation to sustainability with motives of reuse, recycle, DIY etc. Even with this implicit relation, this project is right into this issue because the definition of it is always-already initiated from an existing object. However, as the students were not forced to choose wasted materials or obsolete objects only, it became sometimes problematic to create a consistent scenario about them, like transforming a brand new expensive object into an inferior function as downcycling. On the other hand, this type of freedom in formulation of scenarios made us able to make good quality discussions about the consistency of a project itself, where all the issues concerning recycle, reuse, lifecycle of a product etc. are subjects. This flexibility is also where the variety of different concepts lies as mentioned at the beginning of the paper on the terminology about design. If a project was strictly defined as for example "recycle design" "readymade design" then the clichés or existing examples could have dominated the students. However, starting with a broader brief obviously created a space about the variety in design alternatives and again variety and richness in discussions. Scenario consistency and dominancy also point out the act of design as developing service ideas; the importance of the whole process of demand, use and disposal rather than the product itself.

In brief; functionality, reproducibility, consistency of visual language, consistency of scenario and being designerly can be counted as the general criteria that affect the quality and the assessment of the project.

\section{Presentation of projects}

In this section some of the projects that illustrate the issues about this study in the order of intervention types required in the brief, that are namely emphasize, change and add functions will be presented. Under these headings, we will try to pinpoint the related concerns, key terms and criteria mentioned above. 
Fig. 5 One example of enhance function projects: Wet Wipe Package enhanced
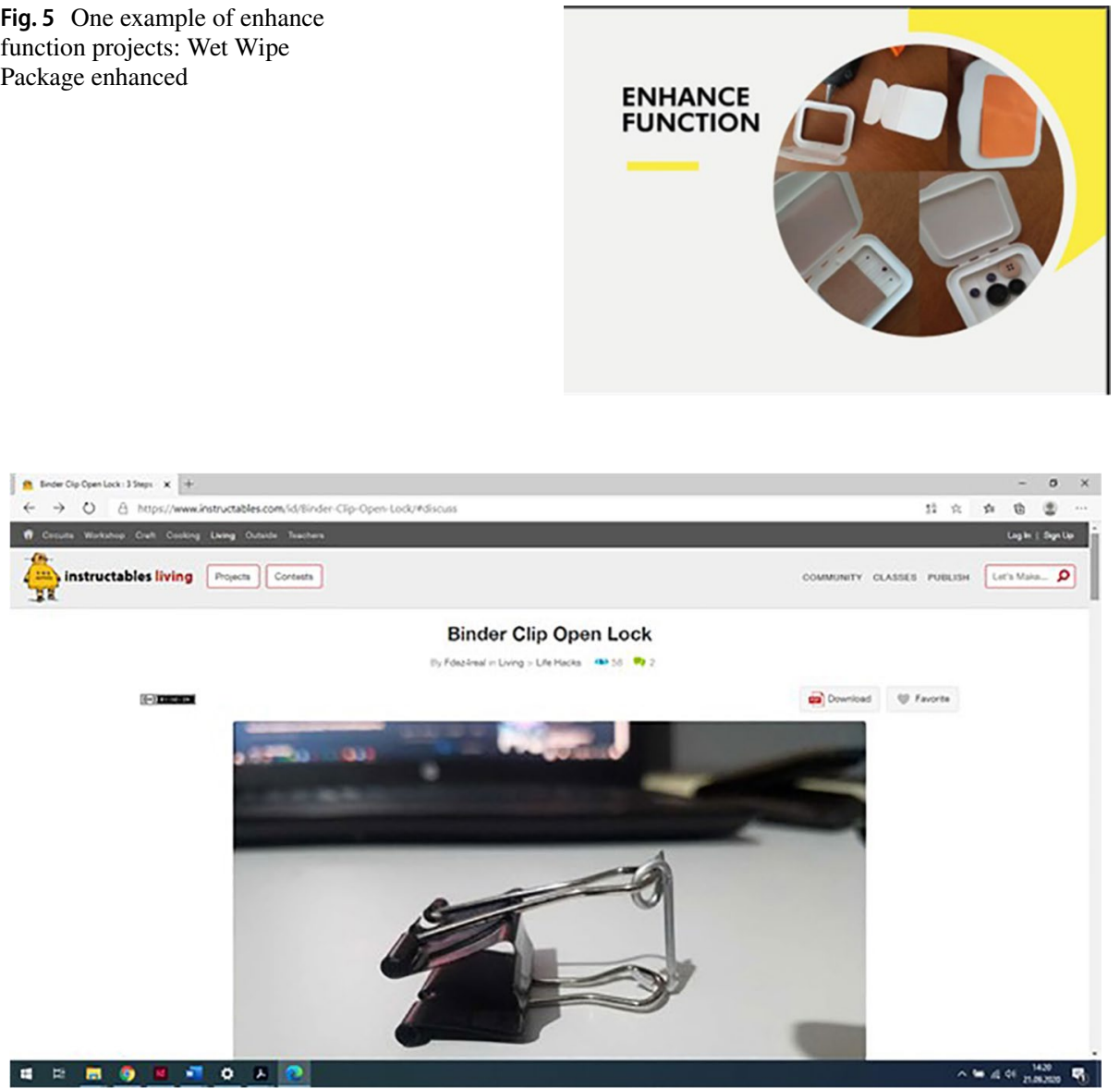

Fig. 6 Enhancing Function: Binder Clip Open Lock

\section{Enhance function}

In this category students tried to keep the existing function of the object they selected and found ways to improve the quality of that function in different and designerly ways. Sometimes they had difficulty intervening in that category because it was easily mixed with the categories of change function or add function in different cases. In such difficulties students were asked to make consistent and convincing definitions about their intent in order to tackle this problem.

In the project in Fig. 5, the student defined her intervention as enhancing function even if the original function of the object is lost because it is a part of an existing package design (wet wipe package) which is normally thrown away after use. However, she made a definition in such a way that the "closing" or "entrapping" function is "emphasized" so that it may be regarded as suitable for this category (Fig. 5).

"Binder Open Lock" (Fig. 6) on the other hand as an intervention which is directly related with the original function suits this category in a much better way. We can say 
Fig. 7 Enhancing function of a jar

Fig. 8 Enhancing function of a jar II
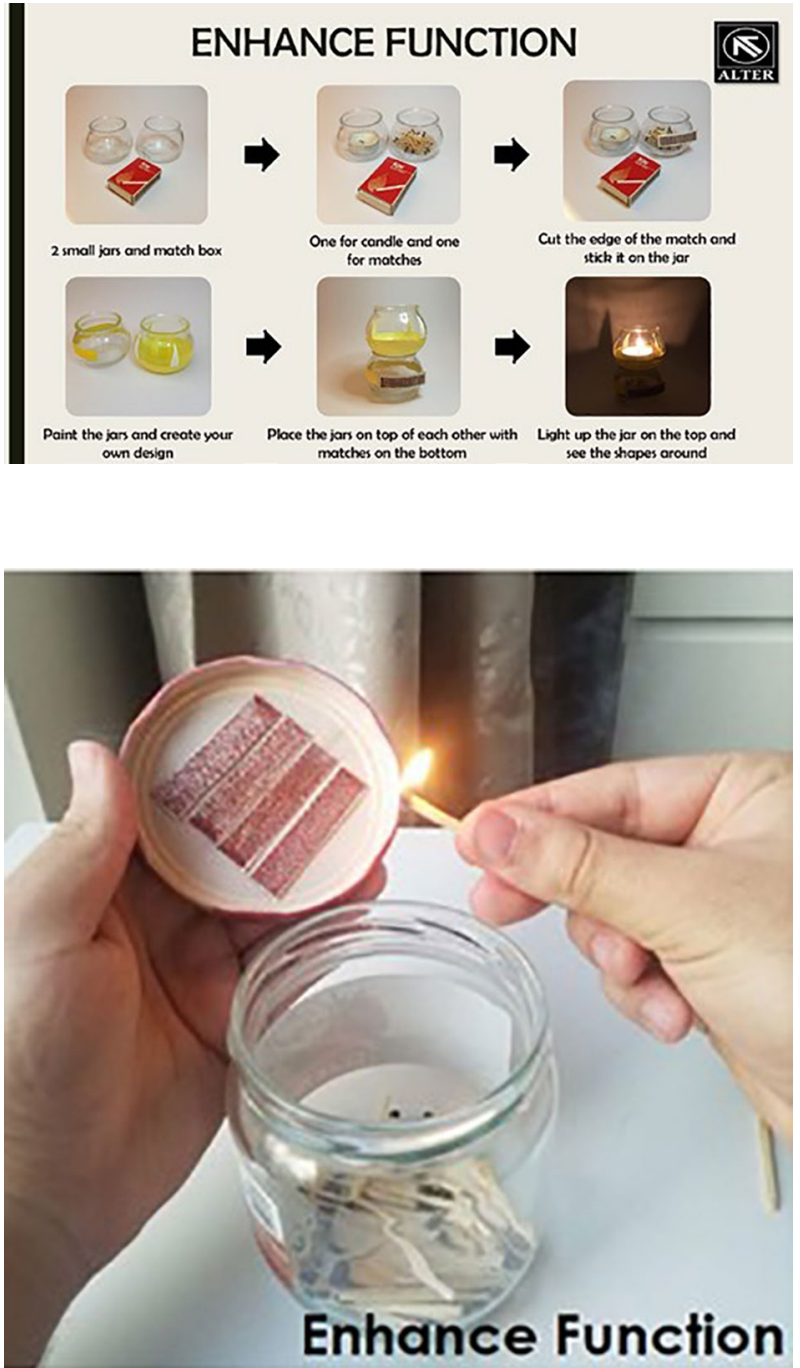

that it makes it easier for the binder to function, which has some pain points already like the extra force needed to stretch the object to open.

This intervention (Fig. 7) is at the cutting edge between the categories of enhance and add function. It is discussed that if the ultimate function of this jar is thought as a candle holder, the function of burning the candle can be regarded as primary. If it is thought in a more generic way as a multi use container, the intervention becomes more like adding a function. So the importance of making good definitions becomes very important to differentiate these categories.

A similar project with a different scenario, not including the candle holder function suggests a different definition, this time using the advantage of closing the cap of a jar for putting out the match fire (Fig. 8). 
Fig. 9 Metallic coat hanger transformed into a toilet paper holder

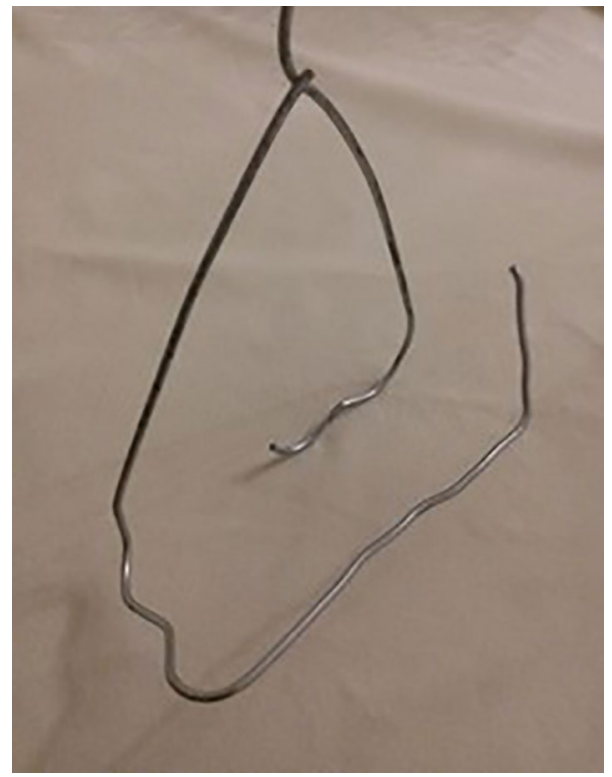

This category was helpful for students to analyse the existing function of the objects, find its problems and try to enhance the function with a simple intervention, also to make discussions about what designerly is. Most of the discussions revolved around topics such as development and betterment of design. It can be said that students had a chance to critique products with a new understanding of problem solving. It was also helpful for students to get used to ideas and processes about evolution of design.

\section{Change function}

Even if it was not stated in the brief, this category of intervention potentially triggers discussions about the terms like hacking, recycle and upcycle as the original function of the object is not kept as it is. However, the existing affordances related to the form, material etc. were asked to be considered and referred to during design.

In the student projects, one of the first models developed for this category is the transformation of a coat hanger into a toilet paper holder. Because the size of the hanger has changed and become smaller than the original, it is obviously not functional anymore as a coat hanger (Fig. 9).

This can be given as an example of one that being designerly as one of the considerations of this project was not achieved. The reasons for this result are discussed in terms of consistency of form language and reproducibility. These types of forms are considered as not properly designed because they don't have a consistent form with a certain identity which makes it characteristic and easy to remember and reproduce. Even if the project can be successful in terms of intervention of an existing product into a new, changed or emphasized function, the degree of being "designerly" was poor because it didn't meet the standards related with above mentioned criteria.

A similar object with the same function transformed again into a similar function with the previous example is worth discussing and comparing here. In this one the intervention 
Fig. 10 Plastic coat hanger transformed into a toilet paper holder
Fig. 11 Scissors used for "change function" category
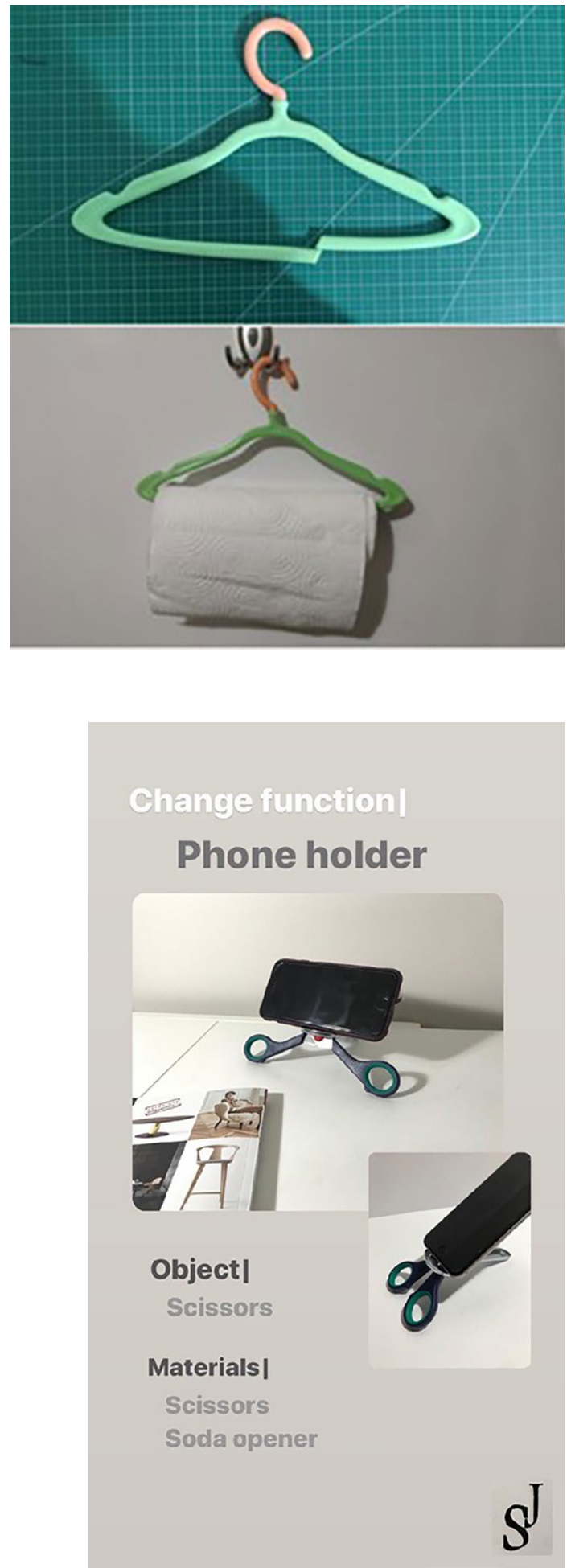
Fig. 12 Food tong made from paper clip and cutlery

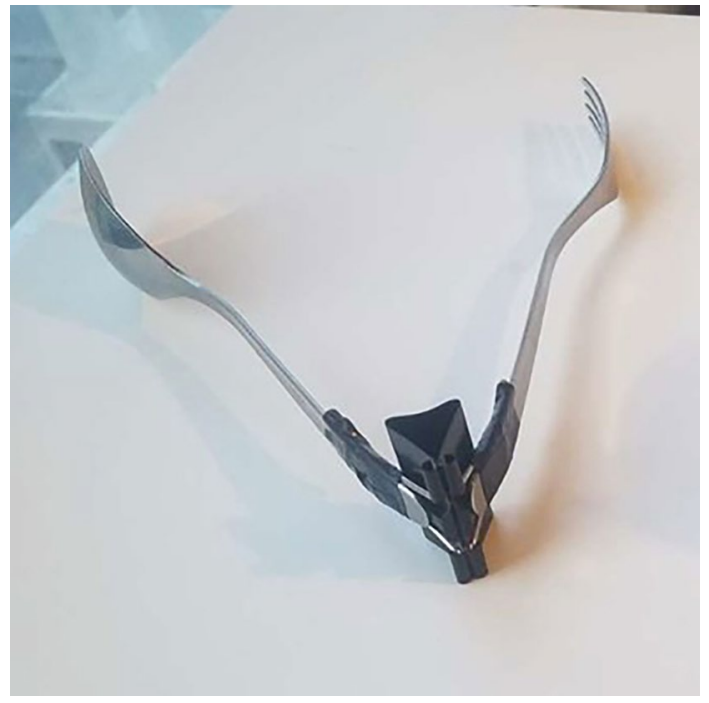

which is a simple crack in a plastic hanger turned the object into a paper towel holder. But this time because of the material chosen and the definability and reproducibility of the intervention, this project became more successful (Fig. 10).

Following piece of design (Fig. 11) is successful in terms of achieving a consistent form in its new formula, together with a consistent scenario where the new use of the combination of the two existing objects -scissors and a classical bottle opener-, because it does not ruin any of them. This aspect is important in this piece because the objects used in this design are not easily consumed and thrown away. Also, the position of scissors allows different positions of the new function which are horizontal and vertical use of smartphones.

"Food Tong" is also a radical change in the function that changes not only the function of the product as a paperclip, but also the context change into a kitchen utensil (Fig. 12).

In this category, consistency of the formulated scenario is so critical especially from the criteria concerning questions such as these; "is the starting point of design is a thrown out material?", "is it a standard product that can be found easily worldwide for the people who want to implement the intervention?", "is it possible to turn it back to the original function when not in use in its second and new function?" etc.

Some of the students, because they do not have the experience of creating a functional product as first year students, started with some ideas that were impossible to function. Instructors needed to remind several times that the focus of this project is to create something that can be produced by other parties and function in its new definition properly. After some examples with this kind of barriers, students have managed to overcome this problem with their new models.

This object (Fig. 13) as an example of an initial step in the design process is transformed for a new function however it is practically not functioning because the necessary technology is not embedded. Similar problems about the issue of functionality existed in the other category; add function.

One other remark to make in this category, however related with general considerations, is again about the scenario built for the new formulation related with the object. In this 
Fig. 13 Jar changed function for a lighting unit

Fig. 14 Book changed function into a pencil case
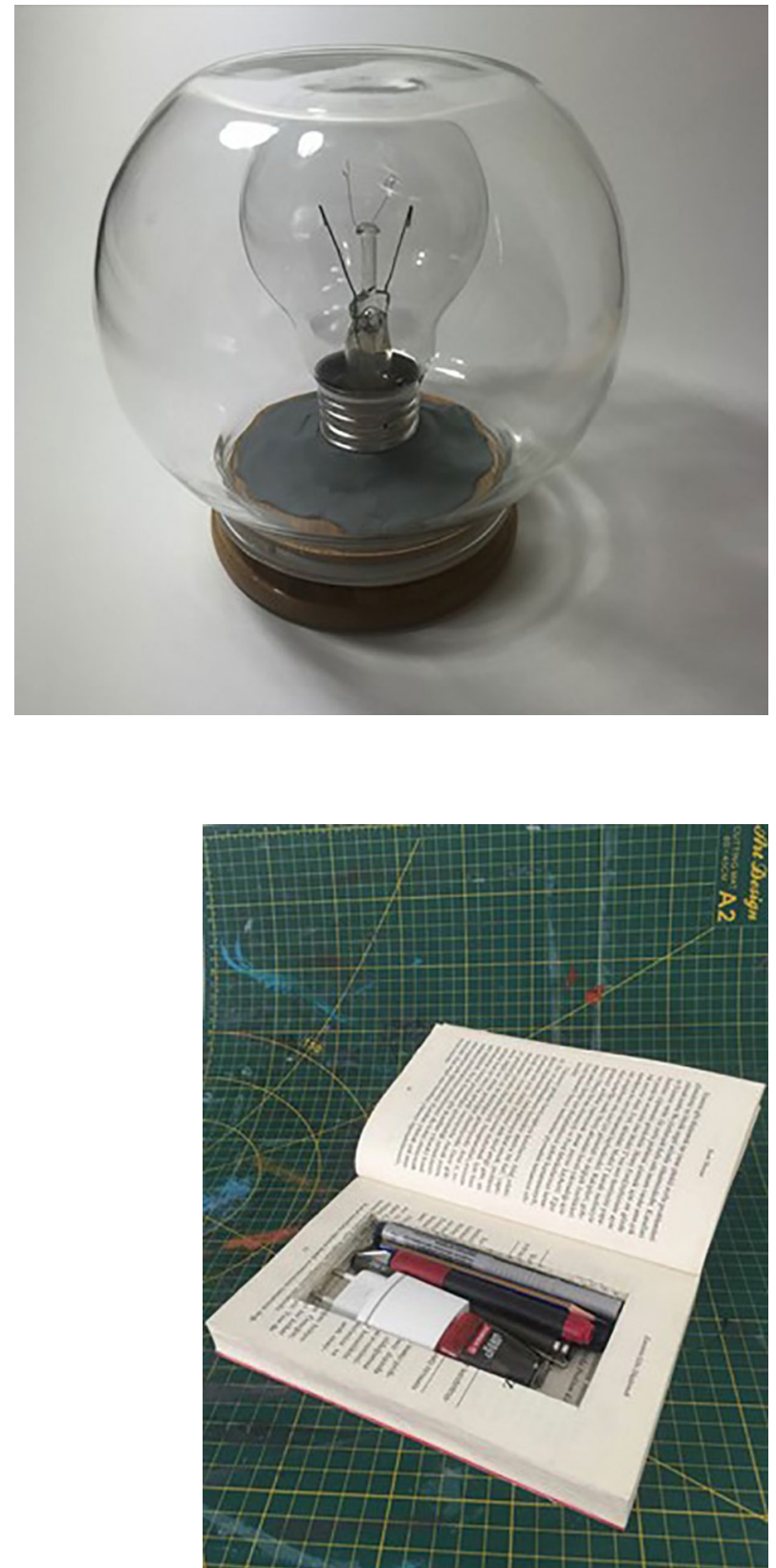

example a book is "ruined" to transform into a hidden case for pen, pencils, and other stuff in a very functional way, but the transformed object triggers a question about ethics (Fig. 14). 
Fig. 15 A jar customized for portioning as an added function

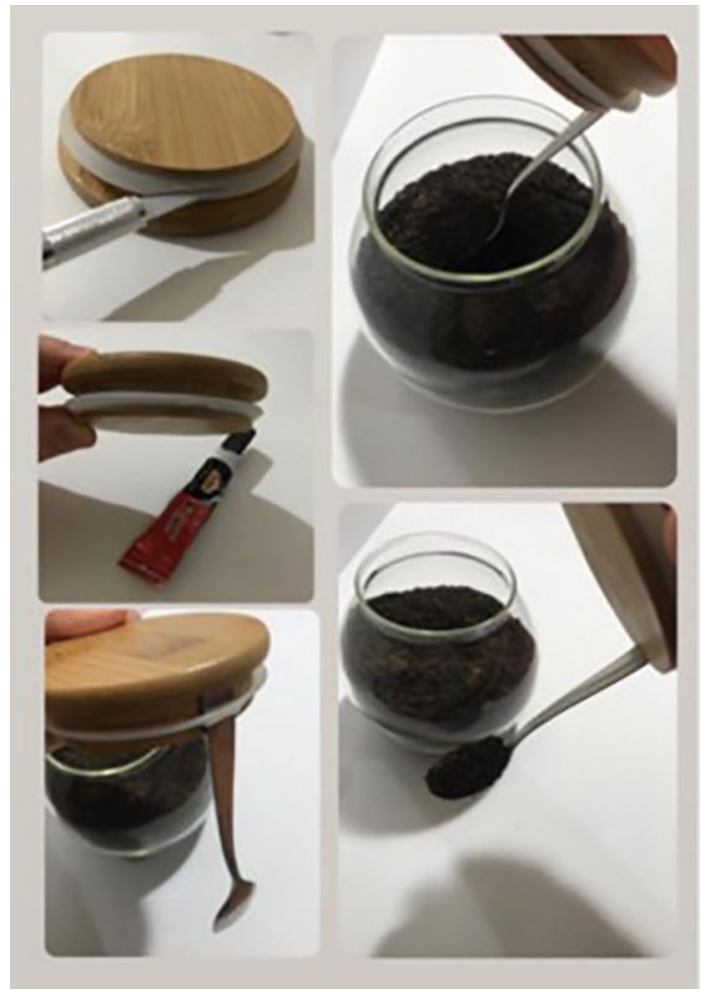

Fig. 16 Wet wipe pack transformed into a phone stand for carrying coins etc

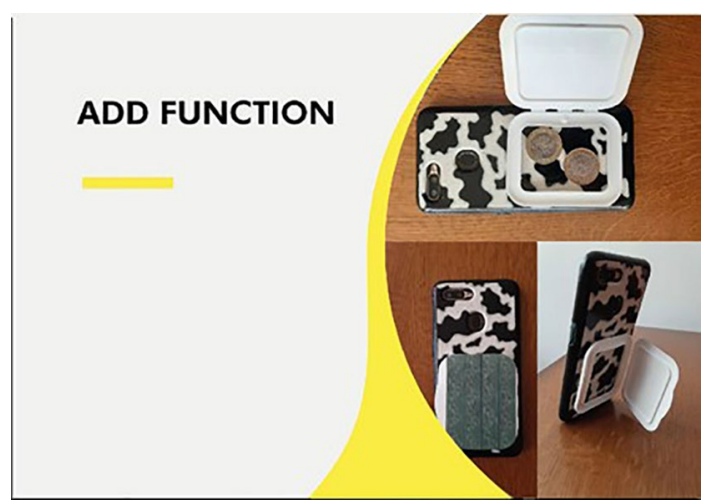

\section{Add function}

In this category students are required to keep the original function of the product they selected and add a function related to it. Just like in the category of "enhance function", being designerly is important in material, scenario and application consistency in "add function". Just like enhancing function, adding function could be a way to improve an existing product if the intervention is applied in a smart and consistent way. 
Fig. 17 Scissors holder made from plastic bottle cap
Fig. 18 Scissors with added function of measuring
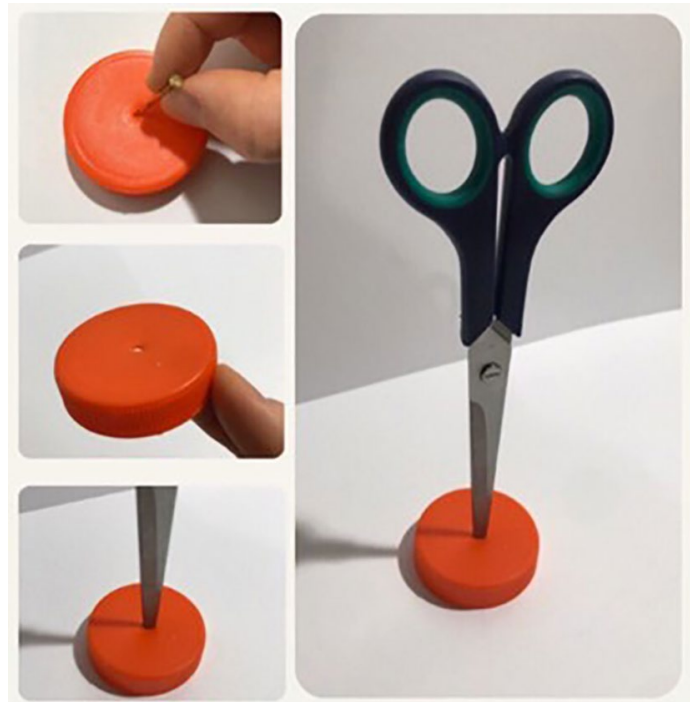

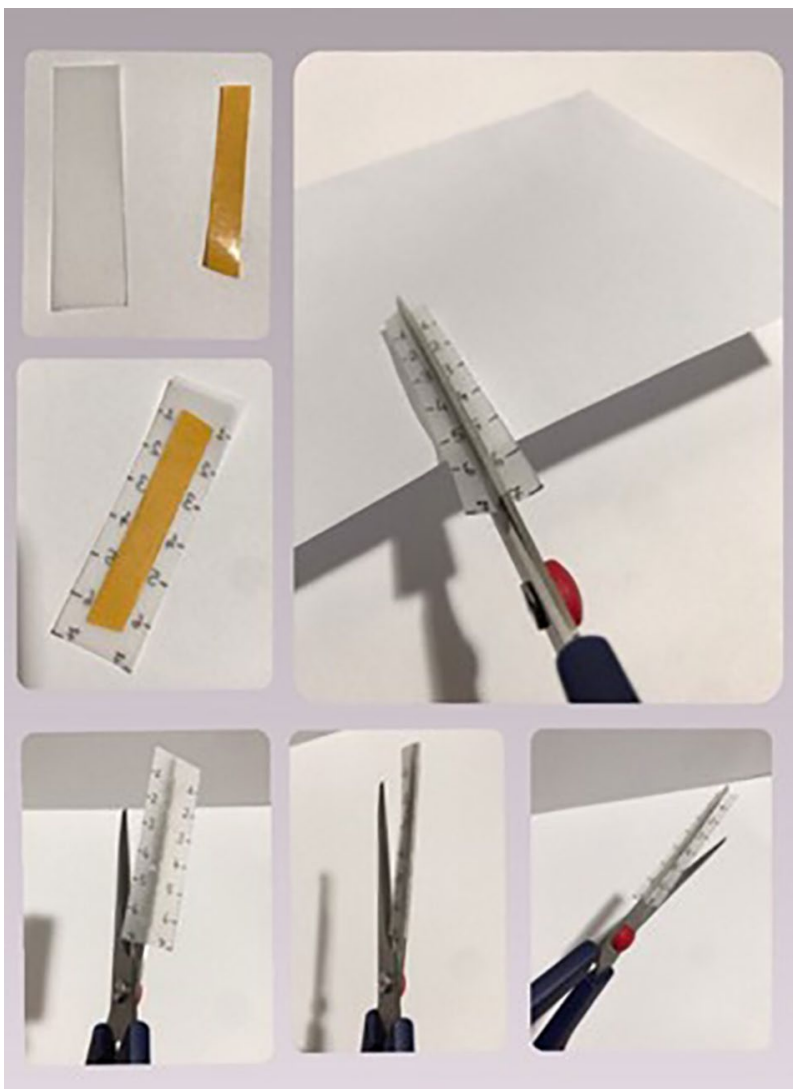


Fig. 19 Lighter with the additional function of cutting and making holes

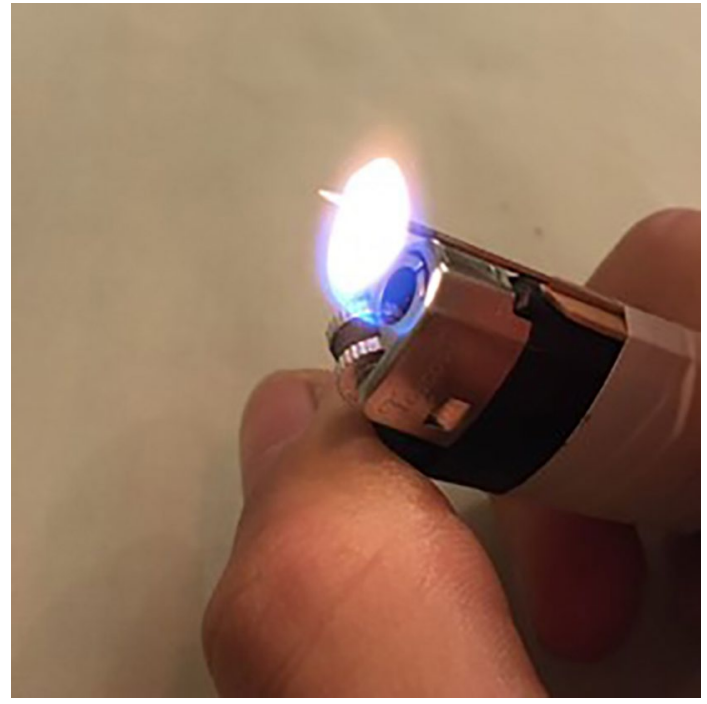

This example is a successful one as an object with an added function, as we can understand or imagine that it can be applied to the existing product without any radical change in the production line, or easily applicable by the user with a simple intervention, i.e. it fulfills the requirement about the reproducibility (Fig. 15).

Next example (Fig. 16) is also suitable for this category because the closing part of a wet wipe pack as a thrown-out material is transformed into a phone stand together with keeping small stuff like coins. In this one, scenario consistency related with sustainability is successful because it upcycles an existing thrown out plastic material.

In this example, on the other hand, creating a stand for a scissors seems not necessary or functional because there are other objects which fulfills this function in a better way like pencil holders that scissors and other similar accessories can be placed in. This can be a good example of not being consistent in a holistic scenario concerning contextual relations (Fig. 17).

Even if it is not working properly, added function to scissors for measuring the length of the cut seemed like a consistent scenario related to the use of the object (Fig. 18).

Another intervention transformed a lighter into a product that can process, i.e. drill and cut plastic material. During the process of the project, the student not only produced, but also used it in his model making processes that can be regarded as a good test about the new function assigned to it (Fig. 19).

\section{Conclusion and discussion}

This study can be regarded as an original experimentation of a terminological model taken from a graduate design research on an undergraduate design project. Regarding the variety of the models, materials and discussions, we can say that it is a successful step taken for design education. We can also say that it constituted a new bridge between graduate and undergraduate design education that could be fruitful and inspiring for building similar formulations in design education curricula. 
One of the achievements of this project was that students learned to look at the "built environment" as a source of design. The main axis between the material choice from scratch and from what is already produced as products is where all the fruitful discussions are placed. To be able to read and intervene on existing objects and structures also open new gates about analyzing material qualities, details, affordances and new associations related with them. These kinds of discussions are also what design students and design professionals should always be careful and sensitive about as cognitive processes including thinking, knowing, remembering, judging, and problem-solving, especially with regards to environmental and technological concerns. These concerns are believed to become instincts in every profession related with production such as design. These instincts or habits are very important to further develop new ideas and strategies for design students in their training, and to create an intellectual difference in their professional lives.

\section{Suggestions for further studies}

The time and context the project is given when the COVID-19 has started to change our lives is significant because of the practical advantage of the project formulation. As it is based on the existing objects at hand, the resources to build models on are more convenient to find and innovate; also the limitations about the materials forced students to be more creative. However, students were reluctant to create ideas about the problems directly related to the pandemic, even if they were encouraged to in the brief. Other project formulations could concentrate on this problem only with related brief ideas.

The fact that it opens a window to other platforms like instructables.com, rather than university design studio as a sterile ecosystem, we can say that students had a chance to enrich their perspectives and challenge their ideas with different parties; also practiced on how to present their ideas in an instructional format as a way to communicate the steps of production. However, in this part of the project it was hard to encourage students to share and challenge their ideas, because it was originally initiated as a school project. But we believe that if students had more time and motivation to collect feedbacks and develop their designs accordingly (it could have been given as another requirement in the process), there would definitely be a chance for them to capture that maker or hacker spirit with the advantage of design education added, in other words being designerly.

Accordingly, there was no time for tutors and students to understand whether "being designerly" was advantageous in a platform like this. Another reason for not being able to understand the effect of this parameter could be that the project was held by freshman design students. If the same brief was given to senior students, the outcome could have been different. For example, a project could be formulated to require students to analyze the hacking strategies in these kinds of platforms and create their own design formulations accordingly or a follow up project to interpret and convert hacking ideas developed here as a starting point for new industrial design projects to be developed and manufactured.

The paper seeks to explore new paths in design education about how social and environmental benefit by design can be increased without any need to highlight related terminology. Because of this formulation, all the parameters or titles referred to in design education like sustainability, eco design, recycle and reuse were discussed in a sincere atmosphere. The consistency of scenario and other design parameters like form 
and materials are made referential with these issues during the process. Trying to build their own definitions, students did not tend to get influenced by existing examples easily achieved by a simple Google search. For further similar research, it could be a good idea to collect reflections of students about this phase of the project as qualitative and quantitative data, rather than presenting and explaining the project examples only.

One other contribution of this study is the checklist of design parameters suggested for evaluating similar projects in design education and platforms where different sets of design pieces are shared. This list can be improved by adding, subtracting or transforming new or existing parameters according to the subject or the platform the students and designers are given and studied.

\section{References}

Archer, B. (1979). The three Rs. Design Studies, 1(1), 18-20.

Blank, S. (2011). Embrace failure to start up success. Nature, 477, 133. https://doi.org/10.1038/477133a

Burnham, S. (2019). Design Hacking: Finding the Truth in Systems. VRMNTR via PublishDrive.

Campbell, C. (2005). The craft consumer: Culture, craft and consumption in a postmodern society. Journal of Consumer Culture, 5(1), 23-42.

Cantamessa, M., Gatteschi, V., Perboli, G., \& Rosano, M. (2018). Startups' roads to failure. Sustainability, 10(7), 2346.

Corner shower caddy: The Grundtal extra long version. (February 13, 2018). Ikeahackers. Retrieved September 23, 2020 from https://www.ikeahackers.net/2018/02/corner-shower-caddy-extra-longgrundtal.html.

Cross, N. (1982). Designerly ways of knowing. Design Studies, 3(4), 221-227.

De Certeau, M. (1988). The practice of everyday life. University of California Press.

Definition of Industrial Design. (2020). retrieved from https://wdo.org/about/definition/.

Hemnes Baby Changing Dresser - IKEA-Hack. (n.d.). Instructables. Retrieved September 23, 2020 from https://www.instructables.com/id/Hemnes-Baby-Changing-Dresser-IKEA-Hack/.

Duman, S. (2020). Integration of Hacking Mindset and Practice to Industrial Design Education. Online Journal of Art and Design, 8(2), 137-149.

Er, H. A., \& Er, Ö. (2003). Two birds with one stone: a combined project of design education and design promotion for small and medium enterprises (SMEs) in Turkey. In Proceedings of the ICSID 2nd Education Conference (pp. 44-46).

Fleischmann, K. (2015). The democratization of design and design learning-how do we educate the next-generation designer. International Journal of Arts \& Sciences, 8, 101-108.

Galloway, A., Brucker-Cohen, Jonah Gaye, L., Goodman, E., \& Hill, D. (2004). Design for hackability. In Proceedings of the Conference on Designing Interactive Systems: Processes, Practices, Methods, and Techniques.

Goellner, M., Garrett, L., Warell, A., Adank, R., \& Parker, T. (2010). 'Design for Desirability'-Postgraduate Collaboration between Academia and SME Industry. In Proceedings of ConnectED 2010, the 2nd International Conference on Design Education.

Hatch, M. (2014). The maker movement manifesto: Rules for innovation in the new world of crafters, hackers, and tinkerers. McGraw-Hill Education.

von Hippel, E. (2006). Democratizing innovation. The MIT Press.

IKEA HACK: Easy Lamps. (n.d.). Instructables. Retrieved September 23, 2020 from https://www.instr uctables.com/id/IKEA-HACK-easy-lamps/.

Intervention (n.d.) In Macmillan Dictionary.com. Retrieved from https://www.macmillandictionary.com/ dictionary/british/intervention.

Art Intervention. (2019). Retrieved from https://www.tate.org.uk/art/art-terms/a/art-intervention.

Darabi, F., \& Clark, M.C. (2012). Developing business school/SMEs collaboration: the role of trust. International Journal of Entrepreneurial Behaviour \& Research, 18, 477-493.

Kuznetsov, S., \& Paulos, E. (2010, October). Rise of the expert amateur: DIY projects, communities, and cultures. In Proceedings of the 6th Nordic Conference on Human-Computer Interaction: Extending Boundaries (pp. 295-304).

Lewis, T. E., \& Thurman, J. (2019). Tinkering and hacking with objects in the art classroom. Art Education, 72(4), 23-28. 
Mota, C. (2011, November). The rise of personal fabrication. In Proceedings of the 8th ACM conference on Creativity and cognition (pp. 279-288).

Polanyi, M. (2009). The tacit dimension. University of Chicago press.

Saakes, D. (2009, October). Big lampan lamps: designing for DIY. In Proceedings of the seventh ACM conference on Creativity and cognition (pp. 403-404).

Schon, D. A. (1984). The reflective practitioner: How professionals think in action. Basic books.

Simon HA (1969) The sciences of the artificial, Cambridge. MA

Smith, C. (2014). Handymen, hippies and healing: social transformation through the DIY movement (1940s to 1970s) in North america. Architectural Histories, 2(1).

Smith, C. H. (2019). Design Interventions - (Prototyping User Experience 2/3). Retrieved from https:// medium.com/@ careyhillsmith/design-interventions-76a8d1827ad7.

Soyupak, O., \& Bagli, H. (2019). Design thinking as a catalyst for technology start-Ups. International Journal of Research in Business and Social Science (2147-4478), 8(4), 59-70.

Toffler, A. (1984). The third wave. Bantam Books.

Tovey, M. (2015). Chapter 2: design education as the passport to practice. In M. Tovey (Ed.), Design pedagogy: Developments in art and design education. Routledge.

Tüfek, T.E. (2019) Towards Understanding Hacking: A Fieldwork Concerning its Relationship with Product Design (Unpublished master's thesis) Istanbul Technical University, İstanbul, Türkiye

Uluoğlu, B. (2000). Design knowledge communicated in studio critiques. Design Studies, 21(1), 33-58.

von Busch, O., \& Palmås, K. (2006). Abstract hacktivism: The making of a hacker culture. Open Mute.

Wennberg, K., Pathak, S., \& Autio, E. (2013). How culture moulds the effects of self-efficacy and fear of failure on entrepreneurship. Entrepreneurship \& Regional Development, 25(9-10), 756-780.

Wolf, M., \& McQuitty, S. (2011). Understanding the do-it-yourself consumer: DIY motivations and outcomes. AMS Review, 1(3-4), 154-170.

Publisher's Note Springer Nature remains neutral with regard to jurisdictional claims in published maps and institutional affiliations. 